\title{
Asymptotic distribution of the quintile share ratio estimator
}

\author{
Tchilabalo Abozou Kpanzou* \\ Université de Kara, Faculté des Sciences et Technique, BP 43, Kara, Togo
}

Received 29 August 2014; Accepted 16 October 2014

Copyright (C) 2014, Afrika Statistika. All rights reserved

\begin{abstract}
The quintile share ratio (QSR) is a recently introduced measure of income inequality, also forming part of the European Laeken indicators which cover four important dimensions of social inclusion (financial poverty, employment, health and education). In 2001, the European Council decided that income inequality in the European Union member states should be described using a number of indicators including the QSR. From the definition of the QSR, a (traditional) nonparametric estimator for it follows easily as a plug-in-type estimator. However, not much is known about the theoretical properties of this estimator. In this paper the estimator is defined and its asymptotic distribution theory derived. Using a simulation study, some finite sample properties of the limiting normal distribution are explored and reported on.
\end{abstract}

Résumé. Le rapport des quintiles (QSR) est une mesure d'inégalité introduite récemment et fait partie des indicateurs européens couvrant quatre dimensions importantes de l'inclusion sociale, à savoir, la pauvreté, l'emploi, la santé et l'éducation. En 2001, le Conseil Européen a décidé que l'inégalité du revenu dans les pays membres de l'Union Européenne devrait être décrite par un certain nombre d'indicateurs y compris le QSR. De part sa définition, on obtient facilement un estimateur de substitution pour cette mesure d'inégalité. Cependant, très peu est connu sur les propriétés théoriques dudit estimateur. Dans cet article nous définissons l'estimateur nonparamétrique du QSR et nous donnons sa distribution limite. A travers des simulations, nous explorons la loi limite ainsi obtenue, à savoir la loi normale.

Key words: Measures of inequality; Extreme value index; Influence function; Empirical process; Confidence intervals; Simulation.

AMS 2010 Mathematics Subject Classification : 60G70; $62 \mathrm{G} 32$.

${ }^{*}$ Corresponding author Tchilabalo Abozou Kpanzou: kpanzout@gmail.com 
T. A. Kpanzou, Afrika Statistika, Vol. 9, 2014, pages 659-670. Asymptotic distribution of the quintile share ratio estimator.

\section{Introduction}

Measures of inequality, best known in economics, have applications in many other branches of science, e.g. in ecology (see e.g. Magurran, 1991), sociology (see e.g. Allison, 1978), demography (see e.g. White, 1986) and information science (see e.g. Rousseau, 1993). Over the years a large number of such measures has been proposed, with the Gini index perhaps the most well-known one. The Gini index has been studied extensively and its properties documented in a number of papers (see e.g. the survey papers Xu, 2004 and Langel and Tillé; 2013). Indices, most notably the Zenga index, have recently been explored from various points of view; for example finite and asymptotic variance cases have been considered in Greselin et al. (2009), (2010), (2013) and (2014). Another trend, somewhat different from Zengaâ's but equally interesting, which is based on the Palma index, is considered in e.g. Cobham and Sumner (2013a) and (2013a).

A measure of inequality traditionally less well-known is the quintile share ratio (QSR). In recent years, however, it has become more well-known and popular especially since the decision by the European Union in 2001 to include it in its laeken indicators as one of two indicators of inequality, the other being the Gini index. As the ratio of two L-functions, the QSR can be viewed as a function of two parameters, and thus its estimator can be viewed as the function of two L-estimators, or L-statistics. Hence, via a standard technique, known as the (multivariate) delta-method, asymptotic results from those are obtained for the bivariate L-estimator. Such results in finite and infinite variance cases have recently been explored in actuarial and econometric contexts (see e.g. Brazauskas et al. 2007, 2009, and Necir and Zitikis, 2011).

Compared to e.g. the Gini index, relatively little research is available on the statistical properties of estimators of the QSR. In a recent paper Lange and Tillé (2011) discussed the estimation and the variance estimation for the QSR in a complex sampling design framework. The authors build and improve on earlier work by Osier (2006) and Osier (2009). As is to be expected from its definition, the influence function of the QSR is unbounded. The form of its influence function has recently been derived by Kpanzou (2013). In Hulliger and Schoch (2009) robust estimators for the QSR are developed in order to decrease the sensitivity to outlying observations. In this paper we derive the asymptotic distribution of a nonparametric plug-in estimator for the QSR.

Let $X$ denote the random variable of interest, defined on the positive real line, having an absolutely continuous distribution function $F$ with corresponding density function $f$. The quantile function of $F$ is denoted by $Q$, i.e. $Q=F^{-1}$. For given numbers $0<\alpha<\beta<\frac{1}{2}$, define the ratio

$$
\eta \equiv \eta(\alpha, \beta)=\frac{\int_{Q(\beta)}^{\infty} x d F(x)}{\int_{0}^{Q(\alpha)} x d F(x)}=\frac{\int_{\beta}^{1} Q(u) d u}{\int_{0}^{\alpha} Q(u) d u}
$$

The QSR is then given by $\eta(0.2,0.8)$. In what follows we will consider the more general quantity $\eta(\alpha, \beta)$. Results for the QSR will follow directly from that. Let $X_{1}, X_{2}, \ldots, X_{n}$ be a sample of size $n$ on $X$ with the corresponding order statistics denoted by $X_{1, n} \leq X_{2, n} \leq$

Journal home page: www.jafristat.net 
$\ldots \leq X_{n, n}$. A nonparametric plug-in estimator for $\eta(\alpha, \beta)$ is given by

$$
\eta_{n} \equiv \widehat{\eta}(\alpha, \beta)=\left(\frac{1}{n} \sum_{i=[n \beta]+1}^{n} X_{i, n}\right) /\left(\frac{1}{n} \sum_{i=1}^{[n \alpha]} X_{i, n}\right)
$$

Our interest is in the asymptotic distribution of $\sigma_{n}^{-1}\left(\eta_{n}-\eta\right)$, for some suitable sequence of constants $\sigma_{n}$, in the case of heavy-tailed underlying distributions. Depending on the size of the extreme value index (EVI) of the underlying distribution, the limiting distribution is either normal or in the stable class of distributions.

The layout of the paper is as follows. We give the main results in the next section and the proofs in Section 3. A numerical study is reported on in Section 4 and some concluding remarks are given in Section 5 .

\section{Main results}

Note that $\eta_{n}$ is a ratio of two sums of extremes. In the literature a number of results are available on sums of extremes; see e.g. Csőrgo and Mason (1986), Csőrgo et al. (1988a) and (1988b). One should note that statistical inference in finite and infinite variance situations for individual truncated integrals of the quantile function has been developed in a number of papers that deal with a variety of applications. See e.g. Brazauskas et al. (2008), Necir et al. (2010), Necir and Meraghni (2010) for more details. The authors developed strong approximation results for the truncated integrals, which can be automatically coupled to produce bivariate asymptotic results for the pairs of such integrals and thus, in turn, via the delta-method, to obtain desired statistical inference results for ratios and other functions of the two integrals. These results do not however meet the specific requirements of the sums in $\eta_{n}$ in a straightforward fashion. Note also that since $F$ is defined on $(0, \infty)$, the potentially troublesome term in $\eta_{n}$ is the numerator, i.e. the upper sum

$$
\frac{1}{n} \sum_{i=[n \beta]+1}^{n} X_{i, n}
$$

We give a result for the upper sum separately since it may be of independent interest. This result is then applied to obtain the asymptotic distribution of $\eta_{n}$. For $0 \leq s<t \leq 1$ write

$$
\sigma^{2}(s, t)=\int_{s}^{t} \int_{s}^{t}(u \wedge v-u v) d Q(u) d Q(v),
$$

where as usual $u \wedge v=\min (u, v)$. In what follows, we will write for notational convenience

$$
U(\beta)=\int_{\beta}^{1} Q(u) d u \text { and } L(\alpha)=\int_{0}^{\alpha} Q(u) d u
$$

and

$$
U_{n}(\beta)=\frac{1}{n} \sum_{i=[n \beta]+1}^{n} X_{i, n} \text { and } L_{n}(\alpha)=\frac{1}{n} \sum_{i=1}^{[n \alpha]} X_{i, n}
$$

Journal home page: www.jafristat.net 
T. A. Kpanzou, Afrika Statistika, Vol. 9, 2014, pages 659-670. Asymptotic distribution of the quintile share ratio estimator.

Thus

$$
\eta(\alpha, \beta)=\frac{U(\beta)}{L(\alpha)} \text { and } \eta_{n}(\alpha, \beta)=\frac{U_{n}(\beta)}{L_{n}(\alpha)} .
$$

Throughout we make the following assumptions on $F$ :

(A1) The underlying distribution $F$ is defined on $(0, \infty)$ and is absolutely continuous with density function $f$.

(A2) $F$ is a heavy-tailed Pareto-type distribution, i.e.

$$
1-F(x)=x^{-1 / \gamma} l_{F}(x), 0<\gamma<1,
$$

with $l_{F}$ slowly varying at infinity.

Remark 1. Note that (1) holds if and only if $Q(1-u)=u^{-1 / \gamma} l_{Q}(u)$, with $l_{Q}$ slowly varying at zero (see e.g. Seneta, 1976 and Beirlant et al., 2004).

Remark 2. Note that $\sigma^{2}(s, 1)<\infty$ for $\gamma<\frac{1}{2}$ and $\sigma^{2}(s, 1)=\infty$ for $\gamma>\frac{1}{2}$. For $\gamma=\frac{1}{2}$ it could happen that $\sigma^{2}(s, 1)=\infty$. Since $\sigma(\beta, 1)$ is involved in the normalising constant in the limiting result, it has to be replaced by $\sigma(\beta, 1-1 / n)$ in the case where we have $\sigma^{2}(\beta, 1)=\infty$.

Theorem 1. Assume (A1) and (A2) hold. Then, as $n \rightarrow \infty$, if $\gamma \leq \frac{1}{2}$,

$$
\frac{\sqrt{n}}{\sigma(\beta, 1-1 / n)}\left(U_{n}(\beta)-U(\beta)\right) \stackrel{D}{\longrightarrow} N(0,1),
$$

and if $\frac{1}{2}<\gamma<1$,

$$
\frac{\sqrt{n}}{\sigma(\beta, 1-1 / n)}\left(U_{n}(\beta)-U(\beta)\right) \stackrel{D}{\longrightarrow} Y_{\gamma},
$$

where $Y_{\gamma}$ is an asymmetric stable law of index $\frac{1}{\gamma}$.

\section{Remark 3.}

1. Note that if $\sigma^{2}(\beta, 1)<\infty$, then $\sigma^{2}(\beta, 1-1 / n)$ can be replaced by $\sigma^{2}(\beta, 1)$ in $(2)$.

2. If $Y_{\gamma}$ is an asymmetric stable law of index $\frac{1}{\gamma}$, it has a skewness parameter 1 and shift parameter 0 .

The result of Theorem 1 forms the basis of the next theorem. For $U$ a Uniform $(0,1)$ random variable, define a sequence of constants $\left\{\sigma_{n}^{2}\right\}$ by $\sigma_{n}^{2}=\operatorname{Var}\left(T_{n}\right)$, where

$$
T_{n}=U(\beta) \int_{0}^{\alpha}(s-I(U \leq s)) d Q(s)-L(\alpha) \int_{\beta}^{1-1 / n}(s-I(U \leq s)) d Q(s),
$$

with $I($.$) the indicator function. The following result then holds.$

Theorem 2. Under the assumptions of Theorem 1, if $\gamma \leq \frac{1}{2}$

$$
\frac{\sqrt{n} L^{2}(\alpha)}{\sigma_{n}}\left(\eta_{n}-\eta\right) \stackrel{D}{\longrightarrow} N(0,1),
$$

and if $\frac{1}{2}<\gamma<1$

$$
\frac{\sqrt{n} L^{2}(\alpha)}{\sigma_{n}}\left(\eta_{n}-\eta\right) \stackrel{D}{\longrightarrow} Y_{\gamma}
$$

where $Y_{\gamma}$ is an asymmetric stable law of index $\frac{1}{\gamma}$.

Journal home page: www.jafristat.net 


\section{Remark 4.}

1. Note again that if $\sigma^{2}(\beta, 1)<\infty$, then $1-1 / n$ in $\sigma_{n}^{2}$ can be replaced by 1 .

2. It follows by straightforward calculations that

$$
\begin{aligned}
\sigma_{n}^{2}=L^{2}(\alpha) \sigma^{2}(\beta, 1-1 / n)+U^{2}(\beta) \sigma^{2}(0, \alpha) \\
-2 L(\alpha) U(\beta) \int_{0}^{\alpha} s d Q(s) \int_{\beta}^{1-1 / n}(1-t) d Q(t) .
\end{aligned}
$$

\section{Proofs}

The method of proof for the case $\gamma \leq \frac{1}{2}$ is to use the decomposition below in terms of the uniform empirical process and approximating the latter on a special probability space by a Brownian motion. In the case $\gamma>\frac{1}{2}$, a central limit result from Samorodnitsky and Taqqu (1994) is used. Let $U_{1}, U_{2}, \ldots, U_{n}$ be independent Uniform $(0,1)$ random variables. For each $n>1$, the uniform empirical distribution function is defined as

$$
G_{n}(t)=n^{-1} \sum_{i=1}^{n} I\left(U_{i} \leq t\right) .
$$

Denote the order statistics corresponding to $U_{1}, U_{2}, \ldots, U_{n}$ by $0=: U_{0, n} \leq U_{1, n} \leq \ldots \leq$ $U_{n, n} \leq U_{n+1, n}:=1$. Writing $X_{i, n}=Q\left(U_{i, n}\right), i=1, \ldots, n$, we get for integers $0 \leq m<$ $n-k \leq n$ that

$$
\begin{aligned}
\sum_{i=m+1}^{n-k} X_{i, n}-\mu_{n}=n \int_{m / n}^{1-k / n}\left(s-G_{n}(s)\right) d Q(s)+n \int_{m / n}^{U_{m, n}}\left(s-G_{n}(s)\right) d Q(s) & \\
& +n \int_{U_{n-k, n}}^{1-k / n}\left(s-G_{n}(s)\right) d Q(s)
\end{aligned}
$$

where

$$
\mu_{n}=n \int_{m / n}^{1-k / n} Q(s) d s
$$

Proof. Theorem 1. Applying (6) gives

$$
\begin{aligned}
\sum_{i=[n \beta]+1}^{n} X_{i, n}-n \int_{[n \beta] / n}^{1} Q(s) d s & =n \int_{[n \beta] / n}^{1}\left(s-G_{n}(s)\right) d Q(s) \\
& +n \int_{[n \beta] / n}^{U_{[n \beta], n}}\left(s-G_{n}(s)\right) d Q(s) \\
& =n \int_{\beta}^{1}\left(s-G_{n}(s)\right) d Q(s)+R_{n}(\beta) .
\end{aligned}
$$

Journal home page: www.jafristat.net 
Here

$$
\begin{aligned}
& R_{n}(\beta)= {\left[n \int_{[n \beta] / n}^{1}\left(s-G_{n}(s)\right) d Q(s)-n \int_{\beta}^{1}\left(s-G_{n}(s)\right) d Q(s)\right] } \\
& \quad+n \int_{[n \beta] / n}^{U_{[n \beta], n}}\left(s-G_{n}(s)\right) d Q(s) \\
&=R_{1 n}(\beta)+R_{2 n}(\beta),
\end{aligned}
$$

with

$$
R_{1 n}(\beta)=n \int_{[n \beta] / n}^{1}\left(s-G_{n}(s)\right) d Q(s)-n \int_{\beta}^{1}\left(s-G_{n}(s)\right) d Q(s)
$$

and

$$
R_{2 n}(\beta)=n \int_{[n \beta] / n}^{U_{[n \beta], n}}\left(s-G_{n}(s)\right) d Q(s)
$$

Clearly,

$$
\left|R_{1 n}(\beta)\right| \leq \sup _{0 \leq s \leq 1}\left|s-G_{n}(s)\right| n(Q(\beta)-Q([n \beta] / n))=O_{P}\left(n^{-1 / 2}\right)
$$

Similarly,

$$
\left|R_{2 n}(\beta)\right| \leq \sup _{0 \leq s \leq 1}\left|s-G_{n}(s)\right| n\left(Q\left(U_{[n \beta], n}\right)-Q([n \beta] / n)\right)=O_{P}(1)
$$

giving

$$
R_{n}(\beta)=O_{P}(1)
$$

Also,

$$
n \int_{[n \beta] / n}^{1} Q(s) d s=n \int_{\beta}^{1} Q(s) d s+o\left(n^{1 / 2}\right) .
$$

Thus

$$
U_{n}(\beta)-U(\beta)=\frac{1}{n} \sum_{i=[n \beta]+1}^{n} X_{i, n}-\int_{\beta}^{1} Q(s) d s=\int_{\beta}^{1}\left(s-G_{n}(s)\right) d Q(s)+O_{P}\left(n^{-1}\right) .
$$

Now, on the special probability space of Csőrgo, M., Csőrgo et al. (1986) there exists a sequence of Brownian bridges $\left\{B_{n}\right\}_{n \geq 1}$ such that when $\gamma \leq \frac{1}{2}$,

$$
\frac{\sqrt{n}}{\sigma(\beta, 1-1 / n)}\left(U_{n}(\beta)-U(\beta)\right)=\frac{1}{\sigma(\beta, 1-1 / n)} \int_{\beta}^{1-1 / n} B_{n}(s) d Q(s)+o_{P}(1),
$$

from which (2) in the theorem follows immediately. For the case $\frac{1}{2}<\gamma<1$, note that since

$$
\sqrt{n} \sigma(\beta, 1-1 / n) \sim c n^{\gamma} l_{Q}(1 / n) \text { for some } c>0, \text { as } n \rightarrow \infty,
$$

classical theory gives

$$
\sqrt{n}\left(\frac{1}{n} \sum_{i=1}^{n} X_{i}-\int_{0}^{1} Q(s) d s\right) / \sigma(\beta, 1-1 / n) \stackrel{D}{\longrightarrow} Y_{\gamma}
$$


using e.g. a special case of Theorem 1.8.1 of Samorodnitsky and Taqqu (1994).

Also,

$$
\frac{\sqrt{n}}{\sigma(0, \beta)}\left(\frac{1}{n} \sum_{i=1}^{[n \beta]} X_{i, n}-\int_{0}^{\beta} Q(s) d s\right) \stackrel{D}{\longrightarrow} N(0,1),
$$

and $\sqrt{n} \sigma(0, \beta) /\left(n^{\gamma} l_{Q}(1 / n)\right) \rightarrow 0$ as $n \rightarrow \infty$, giving (3) in Theorem 1 .

Proof. Theorem 2. Note that for $0<\gamma \leq \frac{1}{2}$, using the decomposition (6), handling the remainder terms as in the proof of Theorem 1, we obtain on the special probability space of Csőrgo, M., Csőrgo et al. (1986) that

$$
L_{n}(\alpha)-L(\alpha)=\frac{1}{n} \sum_{i=1}^{[n \alpha]} X_{i, n}-\int_{0}^{\alpha} Q(s) d s=\int_{0}^{\alpha}\left(s-G_{n}(s)\right) d Q(s)+O_{P}\left(n^{-1}\right) .
$$

Thus

$$
\frac{\sqrt{n}}{\sigma(0, \alpha)}\left(L_{n}(\alpha)-L(\alpha)\right)=\frac{1}{\sigma(0, \alpha)} \int_{0}^{\alpha} B_{n}(s) d Q(s)+o_{P}(1) .
$$

We now combine this result with (2) in Theorem 1 . Note that always

$$
L_{n}(\alpha) \stackrel{P}{\longrightarrow} L(\alpha), \text { as } n \rightarrow \infty,
$$

and therefore by Slutsky and elementary algebra for $0<\gamma \leq \frac{1}{2}$, we have

$$
\begin{aligned}
\frac{\sqrt{n} L(\alpha)^{2}}{\sigma_{n}}\left(\eta_{n}-\eta\right)= & \frac{\sqrt{n}}{\sigma_{n}}\left[L(\alpha)\left(U_{n}(\beta)-U(\beta)\right)\right]\left(1+o_{P}(1)\right) \\
& \quad-\frac{\sqrt{n}}{\sigma_{n}}\left[U(\beta)\left(L_{n}(\alpha)-L(\alpha)\right)\right]\left(1+o_{P}(1)\right) \\
= & \frac{1}{\sigma_{n}} L(\alpha) \int_{\beta}^{1-1 / n} B_{n}(s) d Q(s) \\
& \quad-\frac{1}{\sigma_{n}} U(\beta) \int_{0}^{\alpha} B_{n}(s) d Q(s)+o_{P}(1)
\end{aligned}
$$

using (7) and (8). Equation (4) follows immediately from this. Consider the case $\frac{1}{2}<\gamma<1$. Since

$$
\begin{gathered}
\sqrt{n}\left(L_{n}(\alpha)-L(\alpha)\right)=o_{P}(1) \\
\frac{\sqrt{n} L(\alpha)^{2}}{\sigma_{n}}\left(\eta_{n}-\eta\right)=\frac{\sqrt{n}}{\sigma_{n}} L(\alpha)\left(U_{n}(\beta)-U(\beta)\right)+o_{P}(1) .
\end{gathered}
$$

Since in this case

$$
\sigma^{2}(\beta, 1-1 / n) \longrightarrow \infty
$$

it follows that

$$
\frac{\sigma^{2}(0, \alpha)}{\sigma^{2}(\beta, 1-1 / n)} \longrightarrow 0
$$

and thus

$$
\frac{\sigma_{n}^{2}}{\sigma^{2}(\beta, 1-1 / n)} \longrightarrow L^{2}(\alpha)
$$

Equation (5) then follows directly from Theorem 1. 
T. A. Kpanzou, Afrika Statistika, Vol. 9, 2014, pages 659-670. Asymptotic distribution of the quintile share ratio estimator.

\section{Numerical results}

In this section a small simulation is reported that was carried out in order to explore the behavior of the asymptotic distribution obtained. We consider the cases of two heavy-tailed distributions, namely the Pareto distribution with $\gamma=0.5$ and the Burr distribution with $\gamma=0.25$. We also looked at the performance of the standard normal confidence intervals for the QSR in terms of coverage probabilities $(C P)$ and average confidence interval lengths $(A C I L)$. Four sample sizes were used viz. 500, 1000, 5000 and 10000, and the simulation was run over 1000 repetitions. The sample size is denoted by $n$.

Figures 1 and 2 compare densities of the asymptotic distribution to the standard normal distribution $N(0,1)$. We can see that these graphs confirm the theoretical results proved above despite the slow convergence to normality. Based on the asymptotic variance, $95 \%$ confidence intervals have been constructed from the simulated samples and the $C P$ and $A C I L$ results are given in Table 1, where $S N I, B P I$ and $B T I$ respectively denote the standard normal, the bootstrap percentile and bootstrap $t$ intervals. The values in brackets are the standard errors associated with the corresponding estimates. The fact that such standard errors are small shows the accuracy of the method used. We can see that the characteristics of the confidence intervals are improved with the sample size, with the bootstrap intervals outperforming the standard normal intervals.

Recall that the idea of this paper is to obtain the limiting distribution of the nonparametric estimator for the QSR and not specifically to carry out inferences on QSR. The simulation was simply used to illustrate how the asymptotic variance could be used to obtain an approximate confidence interval for the QSR. The corresponding results are improved by semi-parametric methods developed in Kpanzou (2011) and Kpanzou et al. (2013).

\section{Concluding remarks}

In this paper we derived the limiting asymptotic distribution function of the nonparametric estimator of the quintile share ratio and we used the asymptotic variance to construct confidence intervals. The performance of such intervals is studied in terms of coverage probabilities and average confidence interval lengths. Although the aim is not to compare the performance of various confidence intervals, a comparison with the bootstrap percentile and bootstrap $t$ intervals shows a better performance of bootstrap methods over the standard normal ones.

Note that the nonparametric estimation method can be heavily influenced by the tails of the underlying distribution, making it sensitive to some data points in such tails. In fact, for a given functional (e.g. QSR), if its influence function is unbounded then it is likely to be sensitive to outliers in the data. This is often the case for measures of inequality (see e.g. Cowell and Flachaire, 2007). Semi-parametric estimators have been developed and shown to be less sensitive to contaminations (see Kpanzou, 2011, Kpanzou et al.; 2013).

Acknowledgements: I would like to thank Prof. T. de Wet and Prof. D. M. Mason for their great contribution to this research.

Journal home page: www.jafristat.net 
T. A. Kpanzou, Afrika Statistika, Vol. 9, 2014, pages 659-670. Asymptotic distribution of the quintile share ratio estimator.
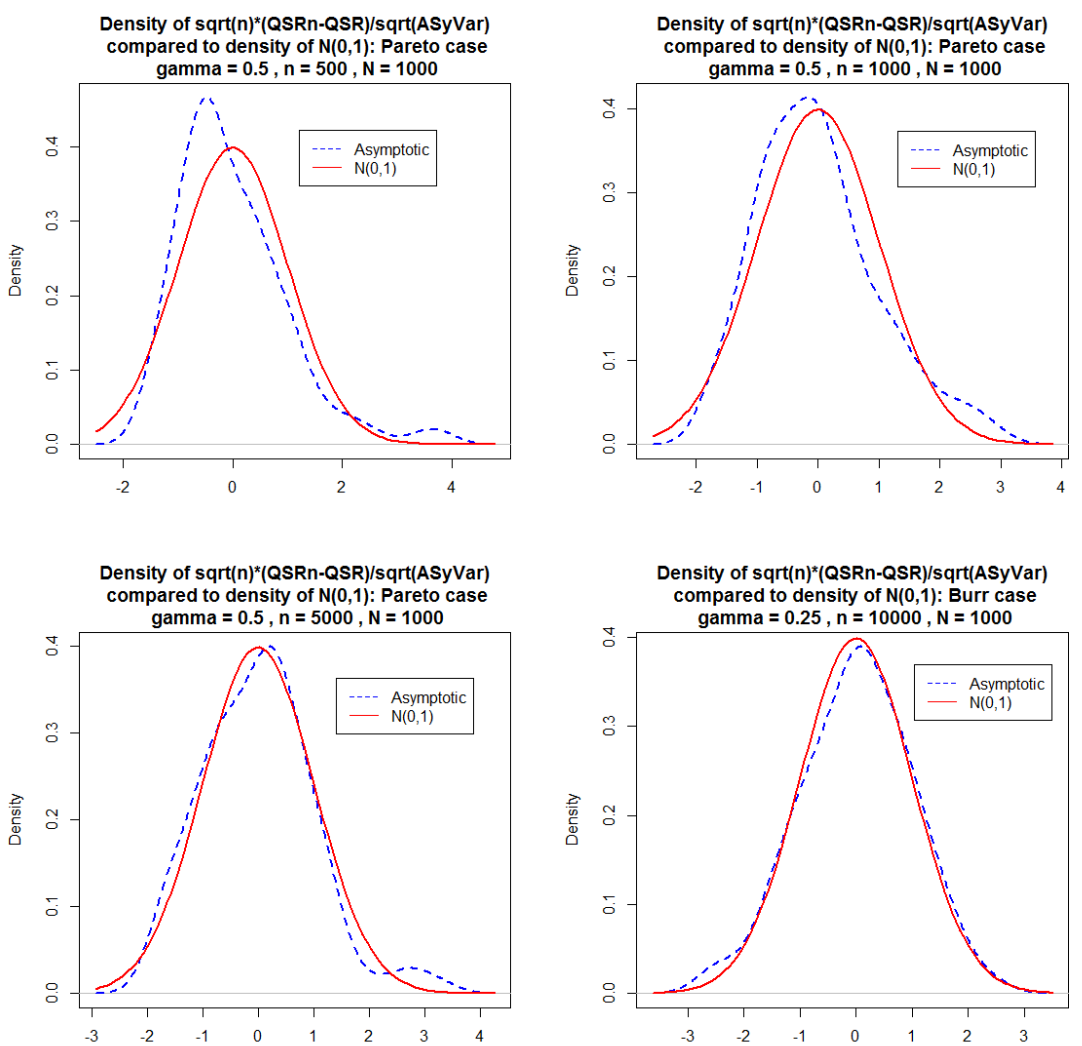

Fig. 1. Comparison of densities of the asymptotic distribution to $N(0,1)$ for samples from the Pareto distribution.

\section{References}

Allison, P.D., 1978. Measures of inequality. American Sociological Review. 43, 478-484.

Beirlant, J., Goegebeur, Y., Segers, J. and Teugels, J., 2004. Statistics of Extremes: Theory and Applications, Wiley.

Brazauskas, V., Jones, B.L., Puri, M.L. and Zitikis, R., 2008. Estimating conditional tail expectation with actuarial applications in view. Journal of Statistical Planning and Inference, 138(11), 3590-3604.

Brazauskas, V., Jones, B.L. and Zitikis, R., 2007. Robustification and performance evaluation of empirical risk measures and other vector-valued estimators. International Journal of Statistics, 65(2), 175-199.

Brazauskas, V., Jones, B.L. and Zitikis, R., 2009. Robust fitting of claim severity distributions and the method of trimmed moments. Journal of Statistical Planning and Inference, 139(6), 2028-2043. 
T. A. Kpanzou, Afrika Statistika, Vol. 9, 2014, pages 659-670. Asymptotic distribution of the quintile share ratio estimator.
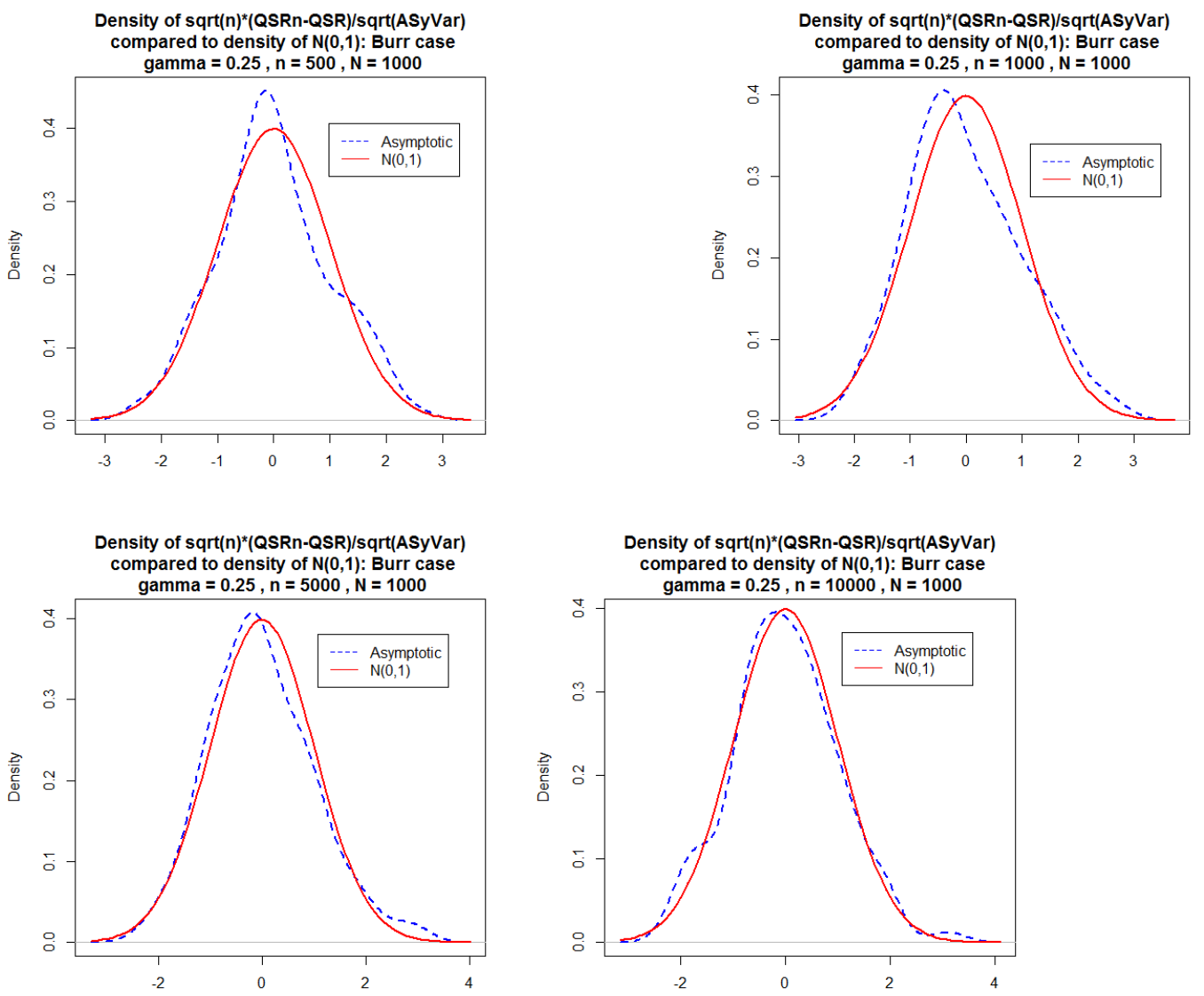

Fig. 2. Comparison of densities of the asymptotic distribution to $N(0,1)$ for samples from the Burr distribution.

Cobham, A. and Sumner, A., 2013. Putting the Gini back in the bottle? "The Palma" as a policy-relevant measure of inequality. Available at the address http://socialprotectionet.org/resources/putting-gini-back-bottle-palma-policy-relevantmeasure-inequality

Cobham, A. and Sumner, A., 2013. Is it all about the tails? The Palma measure of income inequality. Available at the address http://www.ecineq.org/milano/WP/ECINEQ2013308.pdf.

Cowell, F. A. and Flachaire, E., 2007. Income Distribution and Inequality Measurement: The Problem of Extreme Values. Journal of Econometrics. 141, 1044-1072.

Csőrgo, M., Csőrgo, S., Horvath, L. and Mason, D.M., 1986. Weighted empirical and quantile processes. Annals of Probability. 14, 31-85.

Csőrgo, S. and Mason, D.M., 1986. The asymptotic distribution of sums of extreme values from a regularly varying distribution. Annals of Probability. 14, 974-983. 
T. A. Kpanzou, Afrika Statistika, Vol. 9, 2014, pages 659-670. Asymptotic distribution of the quintile share ratio estimator.

\begin{tabular}{|c|c|c|c|c|c|c|c|}
\hline & & & Pareto & & & Burr & \\
\hline $\mathbf{n}$ & & SNI & BPI & BTI & SNI & BPI & BTI \\
\hline \multirow{3}{*}{500} & ACIL & 8.8209 & 8.0263 & 10.6495 & 98.7261 & $\begin{array}{l}87.5276 \\
(0.0307)\end{array}$ & $\begin{array}{l}96.9747 \\
(0.0327)\end{array}$ \\
\hline & $\overline{\mathrm{CP}}$ & 0.9616 & 0.9634 & $\frac{(0.0549)}{0.9647}$ & $\frac{(0.0210)}{0.9624}$ & $\frac{(0.0501)}{0.9639}$ & $\begin{array}{l}0.0321) \\
0.9567\end{array}$ \\
\hline & & $(0.0584)$ & $(0.0532)$ & $(0.0309)$ & $(0.0582)$ & $(0.0529)$ & $(0.0297)$ \\
\hline \multirow{4}{*}{1000} & ACIL & 6.40157 & 5.8249 & 9.4915 & 96.1916 & 63.0785 & 94.7966 \\
\hline & & $(0.0346)$ & $(0.0384)$ & $(0.0510)$ & $(0.0241)$ & $(0.0268)$ & $(0.0286)$ \\
\hline & CP & 0.9575 & 0.9613 & 0.9558 & 0.9611 & 0.9579 & 0.9484 \\
\hline & & $(0.0584)$ & $(0.0532)$ & $(0.0294)$ & $(0.0584)$ & $(0.0532)$ & $(0.0282)$ \\
\hline \multirow{4}{*}{5000} & ACIL & 4.02498 & 3.6624 & 9.0283 & 76.4153 & 37.9516 & 92.338 \\
\hline & & $(0.0240)$ & $(0.0267)$ & $(0.0406)$ & $(0.0176)$ & $(0.0195)$ & $(0.0213)$ \\
\hline & CP & 0.9566 & 0.9526 & 0.9473 & 0.9603 & 0.9551 & 0.9414 \\
\hline & & $(0.0402)$ & $(0.0365)$ & $(0.0292)$ & $(0.0423)$ & $(0.0382)$ & $(0.0285)$ \\
\hline \multirow{4}{*}{10000} & ACIL & 4.00718 & 3.6117 & 8.9062 & 73.5756 & 31.9478 & 82.0122 \\
\hline & & $(0.0221)$ & $(0.0245)$ & $(0.0329)$ & $(0.0166)$ & $(0.0184)$ & $(0.0201)$ \\
\hline & CP & 0.9543 & 0.9518 & 0.9425 & 0.9561 & 0.9514 & 0.9419 \\
\hline & & $(0.0428)$ & $(0.0389)$ & $(0.0287)$ & $(0.0412)$ & $(0.0375)$ & $(0.0282)$ \\
\hline
\end{tabular}

Table 1. Confidence intervals for the QSR based on the NP estimator.

Csőrgo, S., Haeusler, E. and Mason, D.M., 1988. The asymptotic distribution of trimmed sums. Annals of Probability. 16, 672-699.

Csörgo, S., Haeusler, E. and Mason, D.M., 1988. A probabilistic approach to the asymptotic distribution of sums of independent, identically distributed random variables. Advances in Applied Mathematics. 9, 259-333.

Greselin, F., Puri, M.L. and Zitikis, R., 2009. L-functions, processes, and statistics in measuring economic inequality and actuarial risks. Statistics and its Interface. 2(2), 227-245.

Greselin, F., Pasquazzi, L. and Zitikis, R., 2010. Zenga's new index of economic inequality, its estimation, and an analysis of incomes in Italy. Journal of Probability and Statistics. vol. 2010(1), 1-26.

Greselin, F., Pasquazzi, L. and Zitikis, R., 2013. Contrasting the Gini and Zenga indices of economic inequality. Journal of Applied Statistics. 40(2), 282-297.

Greselin, F., Pasquazzi, L. and Zitikis, R., 2014. Heavy tailed capital incomes: Zenga index, statistical inference, and ECHP data analysis. Extremes, 17(1), 127-155.

Hulliger, B. and Schoch, T., 2009. Robust estimation of the quintile share ratio with bias reduction. Presented at the Swiss Statistics Meeting, October 30, 2009, Geneva.

Kpanzou, T.A., 2011. Statistical Inference for Inequality Measures based on Semi-Parametric Estimators. Ph.D. dissertation, Stellenbosch University.

Kpanzou, T.A., 2013. On the Influence Function of the Quintile Share Ratio. To appear in Communications in Statistics - Simulation and Computation, DOI: 10.1080/03610918.2013.788707.

Kpanzou, T.A., de Wet, T. and Neethling, A., 2013. Semi-parametric Estimation of Inequality Measures. South African Statistical Journal. 47, 33-48.

Langel, M. and Tillé, Y., 2011. Statistical inference for the quintile share ratio. Journal of Statistical Planning and Inference. 141, 2976-2985. 
T. A. Kpanzou, Afrika Statistika, Vol. 9, 2014, pages 659-670. Asymptotic distribution of the quintile share ratio estimator.

Langel, M. and Tillé, Y., 2013. Variance estimation of the Gini index: revisiting a result several times published. Journal of the Royal Statistical Society. 176, 521-540.

Magurran, A.E., 1991. Ecological diversity and its measurement, Chapman and Hall.

Necir, A. and Meraghni, D., 2010. Estimating L-Functionals for Heavy-Tailed Distributions and Application. Journal of Probability and Statistics. vol. 2010, 1-35.

Necir, A., Rassoul, A. and Zitikis, R., 2010. Estimating the conditional tail expectation in the case of heavy-tailed losses. Journal of Probability and Statistics, vol. 2010(1), 1-17.

Necir, A. and Zitikis, R., 2011. Coupled Risk Measures and Their Empirical Estimation When Losses Follow Heavy-Tailed Distributions (May 30, 2011). Available at http://dx.doi.org/10.2139/ssrn.1855623.

Osier, G., 2006. Variance estimation: the linearization approach applied by Eurostat to the 2004 SILC operation. Technical Report, Eurostat and Statistics Finland Methodological Workshop on EU-SILC, Helsinki, November 7-8, 2006.

Osier, G., 2009. Variance estimation for complex indicators of poverty and inequality using linearization techniques. Survey Research Methods. 3, 167-195.

Rousseau, R., 1993. Measuring concentration: Sampling design issues as illustrated by the case of perfectly stratified samples. Scientometrics. 28, 3-14.

Samorodnitsky, G. and Taqqu, M.S., 1994. Stable non-Gaussian random processes: stochastic models with infinite variance, Chapman and Hall.

Seneta, E., 1976. Regularly Varying Functions, Springer.

White, M.J., 1986. Segregation and diversity measures in population distribution. Population Index. 52, 198-221.

$\mathrm{Xu}, \mathrm{K} ., 2004$. How has the literature on Giniâ's Index evolved in the past 80 years? Department of Economics, Dalhousie University, Canada. 